\title{
Pre-diagnostic circulating concentrations of insulin-like growth factor-1 and risk of COVID-19 mortality: results from UK Biobank
}

\author{
Xikang Fan ${ }^{1}$. Cheng Yin ${ }^{2}$. Jiayu Wang ${ }^{1}$ Mingjia Yang ${ }^{1} \cdot$ Hongxia Ma ${ }^{1,3,4}$. Guangfu Jin ${ }^{1,3,4} \cdot$ Mingyang Song ${ }^{5,6,7}$. \\ Zhibin $\mathrm{Hu}^{1,3,4} \cdot$ Hongbing Shen ${ }^{1,4}$. Dong Hang ${ }^{1,4}$
}

Received: 23 July 2020 / Accepted: 8 December 2020 / Published online: 9 January 2021

○) Springer Nature B.V. 2021

\begin{abstract}
Coronavirus disease 2019 (COVID-19) deteriorates suddenly primarily due to excessive inflammatory injury, and insulinlike growth factor-1 (IGF-1) is implicated in endocrine control of the immune system. However, the effect of IGF-1 levels on COVID-19 prognosis remains unknown. Using UK Biobank resource, we investigated the association between circulating IGF-1 concentrations and mortality risk (available death data updated on 07 Sep 2020) among COVID-19 patients who had pre-diagnostic serum IGF-1 measurements at baseline (2006-2010). Unconditional logistic regression was performed to estimate the odds ratio (OR) and 95\% confidence intervals (CIs) of mortality. Among 1670 COVID-19 patients, 415 deaths occurred due to COVID-19. Compared to the lowest quartile of IGF-1 concentrations, the highest quartile was associated with a $41 \%$ lower risk of mortality $(\mathrm{OR}=0.59,95 \% \mathrm{CI} 0.41-0.86, P$-trend $=0.01)$. In the continuous model, per 1-standard deviation increment in log-transformed IGF-1 was associated with a $15 \%$ reduction in the risk (intraclass correlation coefficients corrected $\mathrm{OR}=0.85,95 \% \mathrm{CI} 0.73-0.99$ ). The association was largely consistent in the various stratified and sensitivity analyses. In conclusion, our data suggest that higher IGF-1 concentrations are associated with a lower risk of COVID-19 mortality. Further studies are required to determine whether and how targeting IGF-1 pathway might improve COVID-19 prognosis.
\end{abstract}

Keywords IGF-1 · COVID-19 $\cdot$ SARS-CoV-2 $\cdot$ Coronavirus infection $\cdot$ Mortality $\cdot$ UK biobank

\section{Introduction}

An outbreak of Coronavirus Disease 2019 (COVID-19) began in December 2019 and has triggered a public health emergency of international concern [1]. The majority

Xikang Fan and Cheng Yin are co-first authors and contributed equally to this work.

Dong Hang

hangdong@njmu.edu.cn

1 Department of Epidemiology and Biostatistics, International Joint Research Center on Environment and Human Health, Center for Global Health, School of Public Health, Nanjing Medical University, 101 Longmian Avenue, Nanjing 211166, China

2 Department of Laboratory, Jiangning Hospital Affiliated to Nanjing Medical University, Nanjing 211100, China

3 State Key Laboratory of Reproductive Medicine, Center for Global Health, Nanjing Medical University, Nanjing 211166, China of COVID-19 patients have a self-limiting infection and recover, but some suffer severe symptoms and even die [2]. The suddenly deteriorating conditions of some patients are mainly attributed to systematic inflammatory injury caused by the excessive or uncontrolled activation of immune response, known as the cytokine storm, leading to

4 Jiangsu Key Lab of Cancer Biomarkers, Prevention and Treatment, Collaborative Innovation Center for Cancer Medicine, Nanjing Medical University, 101 Longmian Avenue, Nanjing 211166, China

5 Department of Nutrition, Harvard T.H. Chan School of Public Health, Boston, MA 02115, USA

6 Department of Epidemiology, Harvard T.H. Chan School of Public Health, Boston, MA 02115, USA

7 Clinical and Translational Epidemiology Unit and Division of Gastroenterology, Massachusetts General Hospital and Harvard Medical School, Boston, MA 02114, USA 
respiratory distress syndrome (ARDS) and multiple organ failure [3].

Insulin-like growth factor 1 (IGF-1) belongs to the IGF family that plays a critical role in diverse biological activities including cell proliferation, metabolism, differentiation, and survival [4]. Cumulative evidence also supports that IGF-1 pathway can regulate the immune response via interaction with various cytokines (e.g., interferons) and immune cells such as T lymphocytes, macrophages, and bone marrow cells [5]. IGF-1 may act as an important switch controlling the amplitude and quality of the immune response [6]. Of note, IGF-1 administration is already an approved therapy for growth failure and may improve symptoms of autoimmune diseases such as type-1 diabetes and multiple sclerosis [7]. However, whether IGF-1 plays a role in COVID-19 prognosis remains unknown.

Therefore, in the current study, we used the UK Biobank resource, with recently released data on COVID-19 tests and updated death records, to investigate the association between pre-diagnostic serum IGF-1 concentrations and mortality among COVID-19 patients.

\section{Methods}

\section{Study participants}

UK Biobank is a prospective cohort study consisting of approximately half a million participants (aged 37-73 years) recruited across the UK between 2006 and 2010 [8]. Trained nurses collected blood samples from all participants in the assessment centers at baseline and from a subset of approximately 18,000 participants with a repeat assessment between 2012 and 2013. According to the Public Health England database linked to UK Biobank updated on 07 Sep 2020, 1713 patients were diagnosed with COVID-19 among 16,508 participants who had undergone COVID-19 PCR tests. In the current analysis, we also included additional 164 patients who died from COVID-19 according to the National Health Service death records. The patients who had no available data on serum IGF-1 $(n=171)$ or died from other causes $(n=36)$ were excluded, leaving 1670 patients in the final analysis. The included participants had no history of ARDS at baseline.

\section{Assessment of IGF-1}

Details about serum biomarker measurements and assay performances have been described online (http://biobank.ndph. ox.ac.uk/showcase/showcase/docs/serum_biochemistry.pdf). Briefly, serum concentrations of IGF-1 were measured in UK Biobank's purpose-built facility using a 'one step sandwich' Chemiluminescent Immunoassay method based on
DiaSorin Liaison XL Analyzer (Diasorin S.p.A), with a detection range of 1.3-195.0 nmol/L. The average coefficients of variation of IGF-1 derived from internal quality control samples of known high, medium, and low concentrations were $6.18 \%, 5.29 \%$, and $6.03 \%$, respectively. Moreover, the assay of serum IGF-1 was registered with an external quality assurance (EQA) scheme (RIQAS Immunoassay Speciality 1) to verify accuracy. The EQA results showed that $100 \%$ of participated distributions $(n=105)$ were good or acceptable.

\section{Ascertainment of COVID-19 mortality}

Dates and causes of death were obtained from death certificates held by the National Health Service Information Centre (England and Wales) and the National Health Service Central Register Scotland (Scotland) [9]. The outcome of current study was mortality due to COVID-19 (ICD-10 U07) and available death data were updated on 07 Sep 2020.

\section{Ascertainment of covariates}

At baseline, participants attended one of 22 assessment centers across England, Scotland, and Wales where they completed a touch-screen, self-completed questionnaire. Ethnicity, smoking status, and alcohol intake were selfreported. Height, body weight, and waist circumference were measured by trained nurses at baseline, and body mass index (BMI) was calculated as weight in kilograms divided by height in meters squared. Townsend deprivation index was derived from residence using data on car and home ownership, unemployment, and household overcrowding. Physical activity was measured as total metabolic equivalent taskminutes per week for all activity including walking, moderate and vigorous activity.

\section{Statistical analysis}

Because the date of COVID-19 testing did not represent the time of infection, the survival time for each patient could not be accurately estimated, leading to Cox regression models inapplicable in the current analysis. Therefore, we analyzed the association between IGF-1 and COVID-19 mortality using unconditional logistic regression models. Odds ratios (OR) and 95\% confidence intervals $(\mathrm{CI})$ were calculated according to quartiles and continuously per 1-standard deviation (SD) increment in log-transformed IGF-1 concentrations. Model 1 was adjusted for major covariates including age at infection, sex, and ethnicity; Model 2 was further adjusted for Townsend deprivation index, BMI, smoking status, alcohol drinking, physical activity, and prevalent diabetes. We also additionally adjusted for serum glucose, 
cardiovascular risk factors (triglycerides, cholesterol, high density lipoprotein, low density lipoprotein, diastolic blood pressure, systolic blood pressure, cholesterol lowering and antihypertensive medications), and C-reactive protein $(\mathrm{n}=1434)$. We calculated intraclass correlation coefficients (ICC) to assess the reproducibility between the 2 measurements of IGF- 1 available in a subcohort of participants $(n=16,356)$, and then used the ICC value to recalibrate the ORs per 1-SD increment for regression dilution [10]. We also used a restricted cubic spline with 4 knots to explore the dose-response relationship between IGF-1 levels and COVID-19 mortality in Model 2. A likelihood ratio test was used to compare the model with only the linear term of IGF-1 levels to the model with both the linear and the cubic spline terms. $P$ for nonlinear $<0.05$ was considered as nonlinearity, and $P$ for linear $<0.05$ as linearity.

Stratified analyses were conducted according to the median age at infection $(<70, \geq 70$ years), sex (male, female), ethnicity (white, non-white), BMI $(<30, \geq 30 \mathrm{~kg} /$ $\mathrm{m}^{2}$ ), physical activity $(\leq$ median, $>$ median $)$, and smoking status (never, ever) in Model 2. To investigate potential effect modification by these stratification variables, we used a likelihood ratio test comparing the models with and without interaction terms between IGF-1 concentrations and each of the stratification variables.

A sensitivity analysis was performed by excluding participants with baseline cancer or cardiovascular disease. Because IGF-1 is highly related to central obesity, we also did a sensitivity analysis by adjusting for waist circumference instead of body mass index. We used SAS 9.4 for all analyses. All statistical tests were two-sided, and $P<0.05$ was considered statistically significant.

\section{Results}

Among 1670 COVID-19 patients, 415 deaths occurred due to COVID-19. The median time from baseline blood draw to COVID-19 testing was 11.2 years (interquartile range: $10.5-11.9$ years). The distribution of IGF-1 concentrations is shown in Fig. 1, ranging from 4.79 to $58.00 \mathrm{nmol} / \mathrm{L}$, which was similar to the distribution in the whole cohort (data not shown). The mean (SD) value was 21.17 (6.00) in the included COVID-19 patients and 21.40 (5.70) in the rest participants of UK Biobank.

Table 1 summarizes the main characteristics of COVID19 patients according to quartiles of serum IGF-1 concentrations. At baseline, participants with higher IGF-1 had a lower BMI and higher levels of physical activity, and tended to be younger, males, and nonsmokers.

As shown in Table 2, higher IGF-1 concentrations were associated with a reduced risk of COVID-19 mortality in the multivariable models (Model 2: OR comparing quartile 4 versus $1=0.59,95 \% \mathrm{CI} 0.41-0.86, P$-trend $=0.01$ ). In the continuous model, per 1-SD increment in log-transformed IGF-1 was associated with a $15 \%$ lower risk of mortality (Model 2: ICC corrected OR $=0.85,95 \%$ CI 0.73-0.99). The association remained stable after further adjustment for serum glucose, cardiovascular risk factors, and C-reactive protein. In Fig. 2, restricted cubic spline regression analysis suggested an inverse linear relationship between IGF-1 concentrations and COVID-19 mortality $(P$ for nonlinear $=0.95$ and $P$ for linear $=0.049$ ).

Figure 3 shows the forest plot results of stratified analyses. The inverse association between IGF-1 and COVID-19 mortality was largely consistent across subgroups, except for ethnicity and smoking status. The association appeared to be stronger in non-white participants and nonsmokers (both $P$-interaction $<0.05)$.

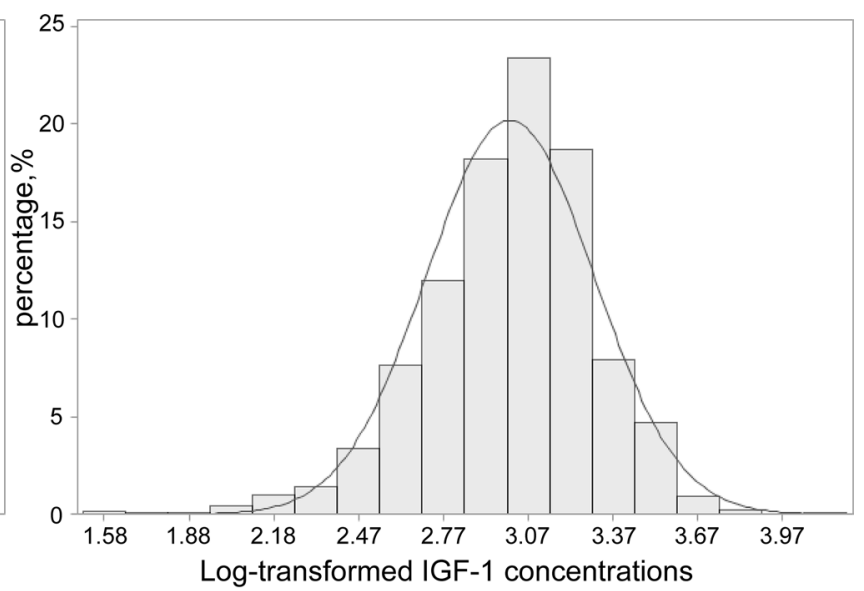

Fig. 1 The distributions of original and log-transformed IGF-1 concentrations 
Table 1 Baseline characteristics of COVID-19 patients according to quartiles of serum IGF-1 concentrations

\begin{tabular}{|c|c|c|c|c|}
\hline \multirow[t]{2}{*}{ Characteristic } & \multicolumn{4}{|c|}{ Serum IGF-1 concentrations (nmol/L) } \\
\hline & Q1 (4.79-17.09) & Q2 (17.09-20.91) & Q3 (20.91-24.51) & Q4 (24.53-58.00) \\
\hline Participants, No & 417 & 418 & 418 & 417 \\
\hline Age at blood draw, mean (SD) (years) & $59.34(7.67)$ & $57.31(9.22)$ & $56.04(9.19)$ & $53.92(9.37)$ \\
\hline Age at infection, mean (SD) (years) & $70.55(7.72)$ & $68.52(9.31)$ & $67.18(9.19)$ & $65.11(9.34)$ \\
\hline Female, No. (\%) & $215(51.56)$ & $201(48.09)$ & $189(45.22)$ & $179(42.93)$ \\
\hline White race, No. (\%) & $368(88.25)$ & $369(88.28)$ & $354(84.69)$ & $363(87.05)$ \\
\hline Prevalent diabetes, No. (\%) & $59(14.15)$ & $35(8.37)$ & $41(9.81)$ & $39(9.35)$ \\
\hline Body mass index, mean (SD) $\left(\mathrm{kg} / \mathrm{m}^{2}\right)$ & $29.91(6.01)$ & $29.10(5.49)$ & $28.29(5.04)$ & $27.70(4.73)$ \\
\hline Physical activity, mean (SD), MET-hours/week & $37.27(38.32)$ & $43.69(44.03)$ & $44.18(41.70)$ & $46.36(48.25)$ \\
\hline Townsend deprivation index, mean (SD) & $0.12(3.50)$ & $-0.21(3.41)$ & $-0.71(3.34)$ & $-0.52(3.33)$ \\
\hline \multicolumn{5}{|l|}{ Smoking status, No. $(\%)^{\mathrm{a}}$} \\
\hline Never & $177(42.45)$ & $194(46.41)$ & $213(50.96)$ & $215(51.56)$ \\
\hline Previous & $178(42.69)$ & $172(41.15)$ & $154(36.84)$ & $144(34.53)$ \\
\hline Current & $55(13.19)$ & $48(11.48)$ & $48(11.48)$ & $55(13.19)$ \\
\hline \multicolumn{5}{|l|}{ Alcohol consumption, No. $(\%)^{\mathrm{a}}$} \\
\hline Daily to three times a week & $142(34.05)$ & $161(38.52)$ & $133(31.82)$ & $138(33.09)$ \\
\hline Twice a week to once a month & $134(32.13)$ & $159(38.04)$ & $165(39.47)$ & $174(41.73)$ \\
\hline Never or special occasions only & $138(33.09)$ & $96(22.97)$ & $119(28.47)$ & $105(25.18)$ \\
\hline
\end{tabular}

MET, metabolic equivalent

${ }^{\text {a }}$ The total did not sum to $100 \%$ because small proportions of participants chose "prefer not to answer"

Table 2 Association between serum IGF-1 concentrations and COVID-19 mortality

\begin{tabular}{|c|c|c|c|c|c|c|c|}
\hline & \multicolumn{4}{|c|}{ Quartile of log-transformed IGF-1 levels, OR $(95 \% \mathrm{CI})^{\mathrm{a}}$} & \multirow[t]{2}{*}{$P$-trend ${ }^{\mathrm{b}}$} & \multirow[t]{2}{*}{ OR per $1-S D$ increment ${ }^{c}$} & \multirow{2}{*}{$\begin{array}{l}\text { Adjusted OR per } \\
\text { 1-SD increment }\end{array}$} \\
\hline & Q1 & Q2 & Q3 & Q4 & & & \\
\hline No. of cases & 142 & 105 & 100 & 68 & & & \\
\hline Model 1 & Ref & $0.70(0.50-0.97)$ & $0.76(0.55-1.07)$ & $0.53(0.37-0.77)$ & 0.001 & $0.84(0.74-0.95)$ & $0.80(0.68-0.94)$ \\
\hline Model 2 & Ref & $0.74(0.53-1.04)$ & $0.86(0.61-1.21)$ & $0.59(0.41-0.86)$ & 0.01 & $0.88(0.78-0.99)$ & $0.85(0.73-0.99)$ \\
\hline $\begin{array}{l}\text { Model } 2 \text { plus } \\
\text { other factors }\end{array}$ & Ref & $0.71(0.49-1.02)$ & $0.90(0.62-1.30)$ & $0.55(0.37-0.83)$ & 0.02 & $0.85(0.74-0.98)$ & $0.81(0.68-0.97)$ \\
\hline
\end{tabular}

OR, odds ratio; $\mathrm{CI}$, confidence interval; ref, reference

${ }^{a}$ Model 1: adjusted for age at infection, sex, ethnicity. Model 2: additionally adjusted for Townsend deprivation index, body mass index, smoking status, alcohol drinking, physical activity, and prevalent diabetes

${ }^{\mathrm{b}} P$-trend was based on the median value of each quartile of log-transformed IGF-1 levels as a continuous variable in the models

${ }^{\mathrm{c}} \mathrm{SD}$ was the standard deviation of log-transformed IGF-1 levels, which was 0.30

${ }^{\mathrm{d}}$ ORs per SD increment were additionally corrected for the intraclass correlation coefficient (0.78) obtained from the subsample of participants with repeat IGF-1 measurements

${ }^{\mathrm{e}}$ Model 2 plus additional adjustment for serum glucose, cardiovascular risk factors (serum lipids, blood pressure, cholesterol lowering and antihypertensive medications), and C-reactive protein. A total of 236 participants had missing data on serum glucose, lipids, or C-reactive protein

Sensitivity analyses showed that the aforementioned association remained basically unchanged by excluding 267 individuals who had baseline cancer or cardiovascular disease (Table 3 ) or by adjusting for waist circumference instead of body mass index (Table 4).

\section{Discussion}

To the best of our knowledge, this study was the first to demonstrate an inverse association between pre-diagnostic 


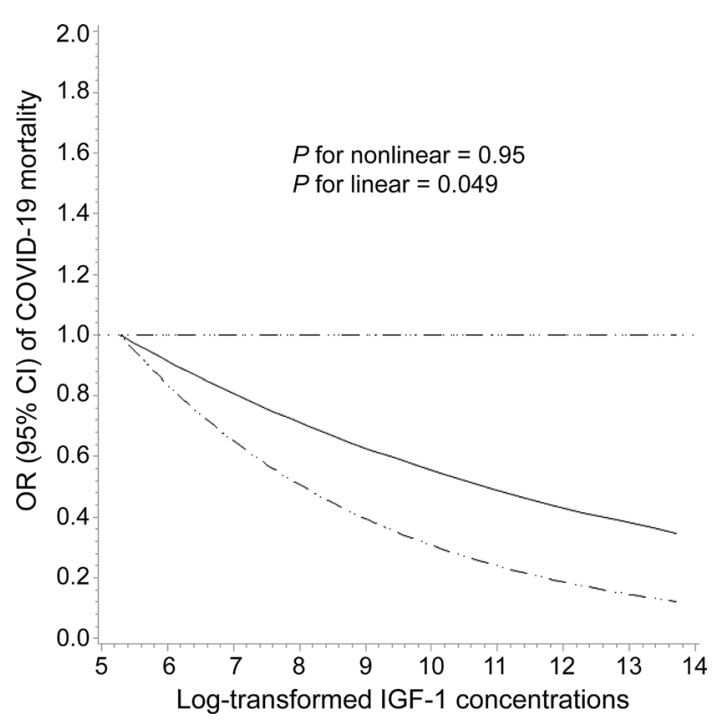

Fig. 2 The dose-response relationship between log-transformed IGF-1 concentrations and COVID-19 mortality according to restricted cubic spline regression analysis. The solid line represents estimates of odds ratios and the dashed lines represent $95 \%$ confidence intervals

circulating levels of IGF-1 and COVID-19 mortality risk among COVID-19 patients. Our findings suggest a potential role of IGF-1 in COVID-19 prognosis and have implications for risk stratification and tailored treatment of COVID-19 patients.

Cytokine storm has been recognized as one of the major causes of ARDS and multiple organ failure in COVID-19 patients. Therefore, effectively suppressing the cytokine storm is important to prevent the disease deterioration and reduce COVID-19 mortality. Markedly elevated levels of circulating interleukin-6 (IL-6), IL-2R, IL-10, tumor necrosis factor- $\alpha$ (TNF- $\alpha)$, etc. have been detected in patients with severe COVID-19 [11, 12]. These cytokines are mainly produced by macrophages and lymphocytes which have been implicated in cytokine storm [13]. Several studies in humans and animal models have suggested an anti-inflammatory effect of IGF-1. For example, there is an inverse relationship between circulating IGF-1 and IL-6 levels [14, 15], and IGF-1/IGF binding protein-3 administration to severely burned patients effectively attenuated inflammatory effects and reduced IL-6 levels [16]. Recombinant human IGF-1 infusion into ApoE-deficient mice significantly decreased macrophage infiltration by downregulating IL- 6 and TNF- $\alpha$ expression [17]. In addition, recombinant human IGF-1 can mediate autoimmune suppression in mouse models of autoimmune disease by increasing regulatory $\mathrm{T}$ cells in affected tissues [7]. These data and our findings shed a light on potential beneficial effects of IGF-1 treatment on COVID19 patients. However, it remains uncertain whether ethnicity and smoking status modify the role of IGF-1 in immune regulation, and other studies also reported that IGF-1 might exert pro-inflammatory effects $[18,19]$. Future studies are necessary to clarify the mechanism of IGF-1 in COVID-19 prognosis and to confirm whether IGF-1 treatment is effective to mitigate the risk of COVID-19 deaths.

Our study has several strengths. First, UK Biobank is a well-designed large cohort and are providing reliable data on COVID-19 diagnosis and related deaths, allowing us to do the analysis in a timely fashion. Second, biochemistry assays of IGF-1 were performed in a single dedicated central laboratory by a standard, reliable method and strict quality control procedures. Third, we were able to adjust for various covariates on demographic and lifestyle factors.

Several potential limitations also need to be acknowledged. First, the observational nature of this study prevents us from inferring causality. However, our sensitivity analyses excluding baseline CVD and cancer supported the robustness of the findings. Second, a single measurement of IGF-1 at baseline may not represent the levels over 10 years. However, the ICC value of 0.78 (95\% CI 0.77-0.79) calculated in a subcohort of UK Biobank, together with previous data [20,21], indicate that IGF-1 levels are generally stable over time. Third, UK biobank enrolled middle and older aged adults only and Caucasians representing the majority of participants, which limited the ability to assess the association among younger people ( $<40$ years) and other ethnicities. Fourth, due to limited coverage of coronavirus testing in the UK, ascertainment bias cannot be avoided. Fifth, recovery and COVID-19 related deaths have been suggested to be competing risk events [22]. However, there is no available data on recovery status for COVID-19 patients in UK biobank, and therefore the current study did not take recovery as a potential competing risk into account. Finally, UK Biobank is not representative of the UK population. However, valid assessment of exposure-outcome associations may be widely generalizable and does not require a representative sample [23].

\section{Conclusions}

The current study indicates that higher serum IGF-1 concentrations are associated with a lower risk of COVID-19 mortality. Further studies are warranted to validate our findings and clarify underlying mechanisms. 
Fig. 3 Forest plots of stratified analysis for the association between serum IGF-1 concentrations and the risk of COVID19 mortality

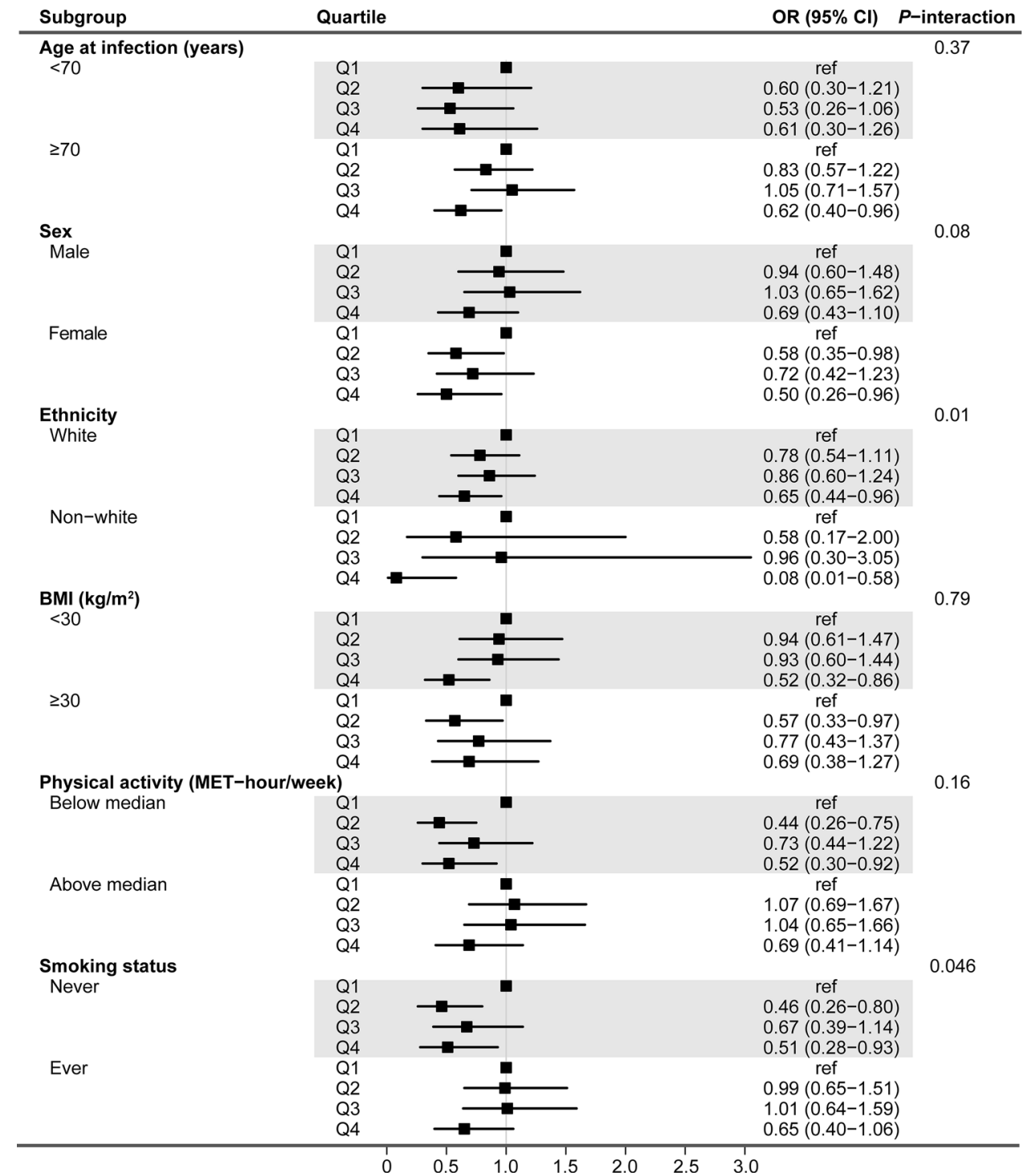

Table 3 Sensitivity analysis for the association between serum IGF-1 concentrations and COVID-19 mortality after excluding 267 participants with baseline cancer or cardiovascular disease

\begin{tabular}{|c|c|c|c|c|c|c|c|}
\hline & \multicolumn{4}{|c|}{ Quartile of log-transformed IGF-1 levels, OR $(95 \% \mathrm{CI})^{\mathrm{a}}$} & \multirow[t]{2}{*}{$P$-trend ${ }^{\mathrm{b}}$} & \multirow[t]{2}{*}{ OR per $1-\mathrm{SD}$ increment ${ }^{\mathrm{c}}$} & \multirow{2}{*}{$\begin{array}{l}\text { Adjusted OR per } \\
\text { 1-SD increment }\end{array}$} \\
\hline & Q1 & Q2 & Q3 & Q4 & & & \\
\hline No. of cases & 113 & 84 & 83 & 44 & & & \\
\hline Model 1 & Ref & $0.73(0.51-1.06)$ & $0.85(0.58-1.23)$ & $0.46(0.30-0.70)$ & 0.002 & $0.80(0.69-0.92)$ & $0.75(0.62-0.90)$ \\
\hline Model 2 & Ref & $0.77(0.53-1.12)$ & $0.94(0.64-1.38)$ & $0.51(0.33-0.80)$ & 0.02 & $0.85(0.73-0.98)$ & $0.81(0.67-0.98)$ \\
\hline $\begin{array}{l}\text { Model } 2 \text { plus } \\
\text { other factors }^{\mathrm{e}}\end{array}$ & Ref & $0.71(0.47-1.08)$ & $1.03(0.68-1.57)$ & $0.57(0.35-0.91)$ & 0.09 & $0.86(0.73-1.01)$ & $0.82(0.66-1.01)$ \\
\hline
\end{tabular}

OR, odds ratio; $\mathrm{CI}$, confidence interval; ref, reference

${ }^{a}$ Model 1: adjusted for age at infection, sex, ethnicity. Model 2: additionally adjusted for Townsend deprivation index, body mass index, smoking status, alcohol drinking, physical activity, and prevalent diabetes

${ }^{\mathrm{b}} P$-trend was based on the median value of each quartile of log-transformed IGF-1 levels as a continuous variable in the models

${ }^{\mathrm{c}} \mathrm{SD}$ was the standard deviation of log-transformed IGF-1 levels, which was 0.30

${ }^{\mathrm{d}}$ ORs per SD increment were additionally corrected for the intraclass correlation coefficient (0.78) obtained from the subsample of participants with repeat IGF-1 measurements

${ }^{\mathrm{e}}$ Model 2 plus additional adjustment for serum glucose, cardiovascular risk factors (serum lipids, blood pressure, cholesterol lowering and antihypertensive medication), and C-reactive protein. A total of 195 participants had missing data on serum glucose, lipids, or C-reactive protein 
Table 4 Sensitivity analysis for the association between serum IGF-1 concentrations and COVID-19 mortality with adjustment for waist circumference instead of body mass index

\begin{tabular}{|c|c|c|c|c|c|c|c|}
\hline & \multicolumn{4}{|c|}{ Quartile of log-transformed IGF-1 levels, OR $(95 \% \mathrm{CI})^{\mathrm{a}}$} & \multirow[t]{2}{*}{$P$-trend ${ }^{\mathrm{b}}$} & \multirow[t]{2}{*}{ OR per $1-\mathrm{SD}$ increment ${ }^{\mathrm{c}}$} & \multirow{2}{*}{$\begin{array}{l}\text { Adjusted OR per } \\
\text { 1-SD increment }\end{array}$} \\
\hline & Q1 & Q2 & Q3 & Q4 & & & \\
\hline No. of cases & 142 & 105 & 100 & 68 & & & \\
\hline Model 1 & Ref & $0.70(0.50-0.97)$ & $0.76(0.55-1.07)$ & $0.53(0.37-0.77)$ & 0.001 & $0.84(0.74-0.95)$ & $0.80(0.68-0.94)$ \\
\hline Model 2 & Ref & $0.74(0.53-1.04)$ & $0.86(0.61-1.21)$ & $0.59(0.40-0.85)$ & 0.01 & $0.88(0.78-0.99)$ & $0.85(0.72-0.99)$ \\
\hline $\begin{array}{l}\text { Model } 2 \text { plus } \\
\text { other factors }\end{array}$ & Ref & $0.71(0.49-1.03)$ & $0.92(0.64-1.33)$ & $0.56(0.37-0.84)$ & 0.02 & $0.86(0.74-0.98)$ & $0.82(0.68-0.98)$ \\
\hline
\end{tabular}

OR, odds ratio; CI, confidence interval; Ref, reference

${ }^{a}$ Model 1: adjusted for age at infection, sex, ethnicity. Model 2: additionally adjusted for Townsend deprivation index, waist circumference, smoking status, alcohol drinking, physical activity, and prevalent diabetes

${ }^{\mathrm{b}} P$-trend was based on the median value of each quartile of log-transformed IGF-1 levels as a continuous variable in the models

${ }^{\mathrm{c}} \mathrm{SD}$ was the standard deviation of log-transformed IGF-1 levels, which was 0.30

${ }^{\mathrm{d}}$ ORs per SD increment were additionally corrected for the intraclass correlation coefficient $(0.78)$ obtained from the subsample of participants with repeat IGF-1 measurements

${ }^{\mathrm{e}}$ Model 2 plus additional adjustment for serum glucose, cardiovascular risk factors (serum lipids, blood pressure, cholesterol lowering and antihypertensive medications), and C-reactive protein. A total of 236 participants had missing data on serum glucose, lipids, or C-reactive protein

Acknowledgements We are grateful to UK Biobank participants. This research has been conducted using the UK Biobank resource under application number 52217.

Author contributions DH was responsible for the conception and design of the study. XF and DH had full access to all the data in the study and took responsibility for the integrity of the data and the accuracy of the data analysis. XF and CY did the statistical analysis and drafted the manuscript. JW, MY, HM, GJ, MS, ZH, and HS critically revised the manuscript for important intellectual content. All authors reviewed and approved the final manuscript.

Funding This study was funded by National Natural Science Foundation of China (82041026, 81820108028, 81521004, and 81973127), National Key R\&D Program of China (2016YFC1000200 and 2017YFC0908300), and Science Foundation for Excellent Young Scholars of Jiangsu (BK20190083). The funders had no role in study design, data collection and analysis, decision to publish, or preparation of the manuscript.

Availability of data and materials UK Biobank is an open access resource. Bona fide researchers can apply to use the UK Biobank data set by registering and applying at http://www.ukbiobank.ac.uk/regis ter-apply.

\section{Compliance with ethical standards}

Conflict of interest The authors have nothing to disclose.

Ethics approval All procedures performed in studies involving human participants were in accordance with the 1964 Helsinki declaration and its later amendments or comparable ethical standards. The ethical approval was obtained from North West Multi-centre Research Ethics Committee (REC reference: 11/NW/03820).

Informed consent Informed consents were obtained from all participants included in the UK Biobank study.

\section{References}

1. Lai CC, Shih TP, Ko WC, Tang HJ, Hsueh PR. Severe acute respiratory syndrome coronavirus 2 (SARS-CoV-2) and coronavirus disease-2019 (COVID-19): The epidemic and the challenges. Int J Antimicrob Agents. 2020;55(3):105924. https://doi.org/10.1016/j. ijantimicag.2020.105924.

2. Azkur AK, Akdis M, Azkur D, Sokolowska M, van de Veen $\mathrm{W}$, Bruggen MC, et al. Immune response to SARS-CoV-2 and mechanisms of immunopathological changes in COVID-19. Allergy. 2020. https://doi.org/10.1111/all.14364.

3. Ye Q, Wang B, Mao J. The pathogenesis and treatment of the 'Cytokine Storm' in COVID-19. J Infect. 2020;80(6):607-13. https://doi.org/10.1016/j.jinf.2020.03.037.

4. Stewart CE, Rotwein P. Growth, differentiation, and survival: multiple physiological functions for insulin-like growth factors. Physiol Rev. 1996;76(4):1005-26. https://doi.org/10.1152/physr ev.1996.76.4.1005.

5. Skarlis C, Nezos A, Mavragani CP, Koutsilieris M. The role of insulin growth factors in autoimmune diseases. Ann Res Hosp. 2019;3:10.

6. Smith TJ. Insulin-like growth factor-I regulation of immune function: a potential therapeutic target in autoimmune diseases? Pharmacol Rev. 2010;62(2):199-236. https://doi.org/10.1124/ pr.109.002469.

7. Bilbao D, Luciani L, Johannesson B, Piszczek A, Rosenthal N. Insulin-like growth factor-1 stimulates regulatory T cells and suppresses autoimmune disease. EMBO Mol Med. 2014;6(11):1423-35. https://doi.org/10.15252/emmm.20130 3376.

8. Collins R. What makes UK Biobank special? Lancet. 2012;379(9822):1173-4. https://doi.org/10.1016/S0140 $-6736(12) 60404-8$

9. Sudlow C, Gallacher J, Allen N, Beral V, Burton P, Danesh J, et al. UK biobank: an open access resource for identifying the causes of a wide range of complex diseases of middle and old age. PLoS Med. 2015;12(3):e1001779. https://doi.org/10.1371/journ al.pmed.1001779.

10. Hankinson SE, Manson JE, Spiegelman D, Willett WC, Longcope C, Speizer FE. Reproducibility of plasma hormone levels in 
postmenopausal women over a 2-3-year period. Cancer Epidemiol Biomark Prev. 1995;4(6):649-54.

11. Chen G, Wu D, Guo W, Cao Y, Huang D, Wang H, et al. Clinical and immunological features of severe and moderate coronavirus disease 2019. J Clin Invest. 2020;130(5):2620-9. https://doi. org/10.1172/JCI137244.

12. Huang C, Wang Y, Li X, Ren L, Zhao J, Hu Y, et al. Clinical features of patients infected with 2019 novel coronavirus in Wuhan. China Lancet. 2020;395(10223):497-506. https://doi.org/10.1016/ S0140-6736(20)30183-5.

13. Arango Duque G, Descoteaux A. Macrophage cytokines: involvement in immunity and infectious diseases. Front Immunol. 2014;5:491. https://doi.org/10.3389/fimmu.2014.00491.

14. Rajpathak SN, McGinn AP, Strickler HD, Rohan TE, Pollak M, Cappola AR, et al. Insulin-like growth factor-(IGF)-axis, inflammation, and glucose intolerance among older adults. Growth Horm IGF Res. 2008;18(2):166-73. https://doi.org/10.1016/j. ghir.2007.08.004.

15. Succurro E, Andreozzi F, Sciacqua A, Hribal ML, Perticone F, Sesti G. Reciprocal association of plasma IGF-1 and interleukin-6 levels with cardiometabolic risk factors in nondiabetic subjects. Diabetes Care. 2008;31(9):1886-8. https://doi.org/10.2337/ dc08-0553.

16. Spies M, Wolf SE, Barrow RE, Jeschke MG, Herndon DN. Modulation of types I and II acute phase reactants with insulin-like growth factor-1/binding protein-3 complex in severely burned children. Crit Care Med. 2002;30(1):83-8. https://doi. org/10.1097/00003246-200201000-00013.

17. Sukhanov S, Higashi Y, Shai SY, Vaughn C, Mohler J, Li Y, et al. IGF-1 reduces inflammatory responses, suppresses oxidative stress, and decreases atherosclerosis progression in ApoE-deficient mice. Arterioscler Thromb Vasc Biol. 2007;27(12):2684-90. https://doi.org/10.1161/ATVBAHA.107.156257.
18. Li G, Zhou L, Zhang C, Shi Y, Dong D, Bai M, et al. Insulin-like growth factor 1 regulates acute inflammatory lung injury mediated by influenza virus infection. Front Microbiol. 2019;10:2541. https ://doi.org/10.3389/fmicb.2019.02541.

19. Wolters TLC, Netea MG, Hermus A, Smit JWA, Netea-Maier RT. IGF1 potentiates the pro-inflammatory response in human peripheral blood mononuclear cells via MAPK. J Mol Endocrinol. 2017;59(2):129-39. https://doi.org/10.1530/JME-17-0062.

20. Borofsky ND, Vogelman JH, Krajcik RA, Orentreich N. Utility of insulin-like growth factor-1 as a biomarker in epidemiologic studies. Clin Chem. 2002;48(12):2248-51.

21. Missmer SA, Spiegelman D, Bertone-Johnson ER, Barbieri RL, Pollak MN, Hankinson SE. Reproducibility of plasma steroid hormones, prolactin, and insulin-like growth factor levels among premenopausal women over a 2- to 3-year period. Cancer Epidemiol Biomarkers Prev. 2006;15(5):972-8. https://doi. org/10.1158/1055-9965.Epi-05-0848.

22. Oulhaj A, Ahmed LA, Prattes J, Suliman A, Alsuwaidi A, AlRifai RH et al. The competing risk between in-hospital mortality and recovery: a pitfall in COVID-19 survival analysis research. medRxiv. 2020:2020.07.11.20151472. https://doi. org/10.1101/2020.07.11.20151472.

23. Fry A, Littlejohns TJ, Sudlow C, Doherty N, Adamska L, Sprosen $\mathrm{T}$, et al. Comparison of sociodemographic and health-related characteristics of UK biobank participants with those of the general population. Am J Epidemiol. 2017;186(9):1026-34. https://doi. org/10.1093/aje/kwx246.

Publisher's Note Springer Nature remains neutral with regard to jurisdictional claims in published maps and institutional affiliations. 